\title{
The Nullstellensatz for real coherent analytic surfaces
}

Fabrizio Broglia and Federica Pieroni

\begin{abstract}
In this paper we prove Hilbert Nullstellensatz for real coherent analytic surfaces and we give a precise description of the obstruction to get it in general. Refering the first, we prove that the ideals of global functions vanishing on analytic subsets are exactly the real saturated ones. For $\mathbb{R}^{3}$ we prove that the real Nullstellensatz holds for real saturated ideals if and only if no principal ideal generated by a function whose zero set is a curve (indeed, a special function) is real. This led us to compare the Nullstellensatz problem with the Hilbert 17th one, also in its weaker form involving infinite sums of squares, proving that they share in fact the same obstruction.
\end{abstract}

\section{Introduction}

If $X$ is a real affine algebraic set and $\mathcal{R}(X)$ denotes its ring of regular functions, it is well known that a prime ideal $\mathfrak{p} \subset \mathcal{R}(X)$ has a regular point in its zero set if and only if its field $k(\mathfrak{p})$ of rational functions can be ordered, that is, $k(\mathfrak{p})$ is a real field and $\mathfrak{p}$ is a real ideal. Moreover, the real algebraic Nullstellensatz states that real ideals are exactly the ideals of all regular functions vanishing on algebraic sets. See for instance [9] and [18].

Analogous results were also proved for rings of real functions where the usual techniques of real algebra apply. Namely, rings of Nash functions and of real analytic function germs at points and compact sets (see [9], [6] and [20]). The situation appears rather different when dealing with rings of global analytic functions without any compactness assumptions.

2000 Mathematics Subject Classification: Primary: 14P15, 14P99; Secondary: 32B10, $11 \mathrm{E} 25$.

Keywords: Nullstellensatz, saturated ideals, sums of squares. 
It remains true that prime ideals with regular points in their zero sets are exactly the ideals vanishing on irreducible global analytic sets (see [5]) but we do not have an algebraic characterization of such ideals and we cannot expect this property to be always satisfied by any real ideal.

A first difference is the existence, already in $\mathcal{O}(\mathbb{R})$, of real prime ideals with empty zero set. For instance, if $\mathfrak{A}$ is an ultrafilter of subsets of $\mathbb{N}$ containing all cofinite subsets, then the ideal $\mathfrak{a}=\{f \in \mathcal{O}(\mathbb{R}) \mid \mathcal{Z}(f) \in \mathfrak{A}\}$ is a real prime ideal in $\mathcal{O}(\mathbb{R})$ with empty zero set (see $[22]$ for a similar example in the complex setting).

Another difference concerns the behaviour of sums of squares. Sums of squares appear quite naturally and at the very beginning, for instance already defining real ideals and the real radical.

Now unlike the algebraic case we can find positive semidefinite functions which can be represented as a sum of infinitely many squares of analytic functions and not as a finite sum (this phenomenon appears already in dimension 3 see [2, Example 5.16]). However, it is clear that if an infinite sum of squares $\sum_{m=1}^{\infty} f_{m}{ }^{2}$ of global analytic functions vanishes on an analytic set, all the functions $f_{m}$ should vanish on it.

Thus, if we look for the class of ideals having the zero property, that is, ideals containing all the functions that vanish on their zero sets, it seems to be reasonable to consider a smaller class of real ideals, at least to remove those real ideals whose zero set is empty and those real ideals, if they exist, that contain infinite sum of squares without containing each addend.

Now, let $\left(X, \mathcal{O}_{X}\right)$ be a real coherent analytic space and denote $\mathcal{O}(X)=$ $H^{0}\left(X, \mathcal{O}_{X}\right)$ its ring of global analytic functions. For any ideal $\mathfrak{a} \subset \mathcal{O}(X)$ let us denote by $\mathfrak{a} \mathcal{O}_{X}$, or shortly by $\mathfrak{a} \mathcal{O}$ when $X$ can be omitted, the coherent sheaf of ideals whose stalk at a point $x \in X$ is $\mathfrak{a} \mathcal{O}_{X, x}$ and consider

$$
\widetilde{\mathfrak{a}}=\left\{f \in \mathcal{O}(X) \mid f_{x} \in \mathfrak{a} \mathcal{O}_{X, x} \text { for any } x \in X\right\}=H^{0}\left(X, \mathfrak{a} \mathcal{O}_{X}\right) .
$$

Clearly

- $\mathfrak{a} \subset \widetilde{\mathfrak{a}}$

- $\mathfrak{a} \subset \mathfrak{b} \Rightarrow \widetilde{\mathfrak{a}} \subset \widetilde{\mathfrak{b}}$

- $\widetilde{\widetilde{a}}=\widetilde{\mathfrak{a}}$.

We say that the ideal $\tilde{\mathfrak{a}}$ is the saturation of $\mathfrak{a}$ and that $\mathfrak{a}$ is saturated if $\mathfrak{a}=\widetilde{\mathfrak{a}}$.

Saturated ideals were first introduced in the complex setting in [16]: they are exactly ideals that are closed with respect to the natural structure of $\mathcal{O}(X)$ as a Frechet space. In [13] the notion of saturated ideals was extended to the real case even though their topological characterization does not work so well anymore, see [14]. 
First of all, notice that a saturated ideal has empty zero set if and only if it is trivial. Moreover, we will see that for a primary ideal the property of being saturated is equivalent to the property of having nonempty zero set (see 2.1).

We recall here that an infinite sum of squares of analytic functions is a series $\sum_{m \geq 1} f_{m}^{2}$ where all $f_{m} \in \mathcal{O}(X)$ such that

(i) the $f_{m}$ 's have holomorphic extensions $F_{m}$ 's all defined in the same complexification of $X$, and

(ii) for every compact set $L, \sum_{m \geq 1} \sup _{L}\left|F_{m}\right|^{2}<+\infty$.

The infinite sum $\sum_{m \geq 1} f_{m}^{2}$ defines well an analytic function on $X$ (see also [1, Definition 1.2]).

This notion leads to a new definition of real ideals that involves also infinite sums of squares. We say that an ideal $\mathfrak{a} \subset \mathcal{O}(X)$ is $\infty$-real if for each (possibly infinite) sum of squares of analytic functions $\sum_{m \geq 1} f_{m}^{2}$ in $\mathfrak{a}$, we have that each $f_{m} \in \mathfrak{a}$. Clearly, an $\infty$-real ideal is in particular a real ideal and then a radical one.

The main property of saturated ideals is the existence of a primary decomposition. Namely, every saturated ideal $\mathfrak{a} \subset \mathcal{O}(X)$ admits a locally finite irredundant decomposition into primary saturated ideals: $\mathfrak{a}=\bigcap_{i} \mathfrak{q}_{i}$ where the primes $\sqrt{\mathfrak{q}_{i}}$ are pairwise distinct and uniquely determined by $\mathfrak{a}$ and $z(\mathfrak{a})=\bigcup_{i} z\left(\mathfrak{q}_{i}\right)$. Moreover, if $\mathfrak{a}$ is a $\infty$-real (resp. real) ideal then any $\mathfrak{q}_{i}$ is a $\infty$-real (resp. real) prime ideal, (see Section 2 for details).

In this paper we mainly study the Nullstellensatz problem for a real coherent analytic surface and for $\mathbb{R}^{3}$.

In the first case we prove the following:

Theorem 1.1. Let $X$ be a real coherent analytic surface. Then, an ideal $\mathfrak{a} \subset \mathcal{O}(X)$ has the zero property, i.e. $\mathcal{J}(\mathcal{Z}(\mathfrak{a}))=\mathfrak{a}$, if and only if it is real and saturated.

This result is a generalization of the real Nullstellensatz for 2-dimensional analytic manifolds (see [10], [4], [7]) and settles some difficulties overlooked in the normal case: we only assume the surface to be coherent.

Also the existence of a primary decomposition allows us to prove the Nullstellensatz for real ideals, not necessarily prime.

In fact real ideals are usually represented as intersection of all real prime ideals containing them, but in this representation real prime ideals with empty zero set may cause some troubles: what we get using primary decomposition is that a saturated real ideal can be written as intersection of real prime ideals each one with not empty zero set. 
On the other hand, note that we cannot deduce a real Nullstellensatz for a general ideal. The main point is that the real radical of a saturated ideal needs not to be a saturated ideal, already in $\mathcal{O}(\mathbb{R})$, as the following example shows.

Example 1.2. Let $f, g \in \mathcal{O}(\mathbb{R})$ :

$$
f(x)=\prod_{n}\left(1-\frac{x}{n^{2}}\right) \quad \text { and } \quad g(x)=\prod_{n}\left(1-\frac{x}{n^{2}}\right)^{n} .
$$

We have that $\mathcal{Z}(f)=\mathcal{Z}(g)=\left\{n^{2} \mid n \in \mathbb{N}^{+}\right\}$. Let $\mathfrak{a}$ be the ideal generated by $g$. Then $\mathfrak{a}$ is a saturated ideal since it is of finite type ([13, Osservazione 1.6.5.c)]) and $\mathfrak{a}=\bigcap_{n}\left(1-\frac{x}{n^{2}}\right)^{n} \mathcal{O}(\mathbb{R})$ is a primary decomposition of $\mathfrak{a}$. Now, if the real Nullstellensatz held for $\mathcal{O}(\mathbb{R})$ there would exist an integer $h \geq 0$, a global analytic function $t \in \mathcal{O}(\mathbb{R})$ and a finite sum of squares $s \in \mathcal{O}(\mathbb{R})$ such that $f^{h}+s=g t$. If we compare orders at the point $(h+1)^{2}$ we achieve a contradiction. Indeed, the left hand side has order $\leq h$ while the right hand side as order $\geq h+1$, which is impossible. This computation shows that $\mathcal{J}(\mathcal{Z}(g)) \neq \sqrt[r]{(g)}$.

Roughly speaking, the problem which appears in the previous example, and in general in the noncompact analytic case, is that the vanishing multiplicity of the function $g$ can grow arbitrarily, while the integer $h$ is fixed, then some boundness conditions is needed. We refer the reader to [19] for this kind of arguments.

For $\mathbb{R}^{3}$ the situation appears quite more complicated. Following [15] we consider special irreducible functions. Roughly speaking, these are functions having zero set of dimension 1 and irreducible holomorphic extension, see Definition 4.1.

Sure, the ideal generated by a special irreducible function cannot have regular points in its zero set, so we would expect it is not a real ideal but this conjecture remains open. However, we prove that this is the only possible obstruction to have a real Nullatellensatz for real saturated ideals. Namely,

Theorem 1.3. Every $\infty$-real (resp. real) saturated ideal in $\mathcal{O}\left(\mathbb{R}^{3}\right)$ has the zero property if and only if no special irreducible function generates a $\infty$-real (resp. real) ideal.

The main point is that any prime ideal for which the zero property fails, turns out to be principal, see Theorem 4.3. In particular, this led us to relate the real Nullstellensatz with the Hilbert 17 th problem at least in $\mathbb{R}^{3}$.

We will say that a nonnegative function $f: X \rightarrow \mathbb{R}$ is an infinite sum of squares of meromorphic functions if there exists $h \in \mathcal{O}(X)$ with $\mathcal{Z}(h) \subset \mathcal{Z}(f)$ 
such that $h^{2} f$ is an infinite sum of squares of analytic functions on $X$ as defined above.

Notice that the classical Hilbert 17th problem can be weakened to ask whether any nonnegative analytic function can be written as a possibly infinite sum of squares of meromorphic functions. This problem for $\mathbb{R}^{3}$ was studied recently and carefully in [15] where the author proves that this Hilbert 17th problem holds for any nonnegative fuction if and only if it holds for any nonnegative special irreducible function. So, in some sense, we prove that these two classical problems have the same kind of obstruction concerning special functions.

Finally we prove some results for higher dimension. Here we are not able to reduce to principal ideals, and so we extend the notion of being special from functions to ideals. A special ideal is a prime ideal $\mathfrak{p}$ such that its complexification $\mathfrak{p} \otimes_{\mathbb{R}} \mathbb{C}$ is also prime and its zero set $Z(\mathfrak{p})$ is contained in the singular locus of $z\left(\mathfrak{p} \otimes_{\mathbb{R}} \mathbb{C}\right)$, that is $\operatorname{dim}_{\mathbb{R}} z(\mathfrak{p})<\operatorname{dim}_{\mathbb{C}} z\left(\mathfrak{p} \otimes_{\mathbb{R}} \mathbb{C}\right)$. Of course the ideal generated by a special function is special.

Thus, in the spirit of Theorem 1.3, we prove that the real Nullstellensatz holds for every real saturated ideal if and only if no special ideal is real.

\section{Preliminaries on saturated ideals}

In what follows, $\left(X, \mathcal{O}_{X}\right)$ will denote a connected paracompact reduced real coherent analytic space. We begin by a classical result concerning primary ideals.

Lemma 2.1. Let $\mathfrak{q} \subset \mathcal{O}(X)$ be a primary ideal.

1. Suppose $\mathbb{Z}(\mathfrak{q}) \neq \emptyset$. Then, a function $f \in \mathcal{O}(X)$ is in $\mathfrak{q}$ if and only if its germ $f_{x}$ is in the stalk $\mathfrak{q} \mathcal{O}_{x}$, for some $x \in \mathcal{Z}(\mathfrak{q})$.

2. The primary ideal $\mathfrak{q}$ is saturated if and only if $\boldsymbol{Z}(\mathfrak{q}) \neq \emptyset$.

Proof. 1. Since $z(\mathfrak{q}) \neq \emptyset$, the "only if" implication is clear. Let us consider the "if" implication. Since $f_{x} \in \mathfrak{q} \mathcal{O}_{x}$ there exist $g_{1}, \ldots, g_{s} \in \mathfrak{q}$ and $a_{1, x}, \ldots, a_{s, x} \in \mathcal{O}_{x}$ such that $f_{x}=\sum_{i=1}^{s} a_{i, x} g_{i, x}$.

Then, the stalk at $x$ of the coherent sheaf $\mathcal{F}=\left(\left(g_{1}, \ldots, g_{s}\right) \mathcal{O}_{X}: f\right)$ coincides with $\mathcal{O}_{x}$. This means that there exists a global section $h \in H^{0}(X, \mathcal{F})$ with $h(x) \neq 0$. Since

$$
h f \in H^{0}\left(X,\left(g_{1}, \ldots, g_{s}\right) \mathcal{O}_{X}\right)=\left(g_{1}, \ldots, g_{s}\right) \mathcal{O}(X) \subset \mathfrak{q}
$$

and no power of $h$ is in $\mathfrak{q}$, because $h_{x}^{k} \notin \mathfrak{q} \mathcal{O}_{x} \subset \mathfrak{m}_{x}$, we get $f \in \mathfrak{q}$. 
Note that a finitely generated ideal $\mathfrak{g}=\left(g_{1}, \ldots, g_{s}\right)$ is saturated: this is an easy consequence of Cartan Theorem B, applied to the natural surjection of coherent sheaves $\mathcal{O}^{s} \rightarrow \mathfrak{g} \mathcal{O}$.

2. The "only if" implication is clear and the "if" implication follows from 1. Indeed, 1 . ensures that

$$
\mathfrak{q}=\left\{f \in \mathcal{O}(X) \mid f_{x} \in \mathfrak{q} \mathcal{O}_{x}\right\}
$$

and [13, Lemma 1.4.2b)] proves that any ideal defined as the "saturation" of a local ideal is saturated.

Next result (cf. [5, Theorem 3.1]) follows straightforwardly from Lemma 2.1.

Theorem 2.2. Let $\mathfrak{q} \subset \mathcal{O}(X)$ be a primary saturated ideal. Assume that there exists a point $x \in \mathcal{Z}(\mathfrak{q})$ such that $\mathcal{J}\left(\mathcal{Z}\left(\mathfrak{q} \mathcal{O}_{x}\right)\right)=\sqrt{\mathfrak{q} \mathcal{O}_{x}}$. Then, $\mathcal{J}(\mathcal{Z}(\mathfrak{q}))=$ $\sqrt{\mathfrak{q}}$ and there exists an integer $h(\mathfrak{q})<+\infty$ such that $(\sqrt{\mathfrak{q}})^{h(\mathfrak{q})} \subset \mathfrak{q}$.

Proof. Take a point $x \in \mathcal{Z}(\mathfrak{q})$ such that $\mathcal{J}\left(\mathcal{Z}\left(\mathfrak{q} \mathcal{O}_{x}\right)\right)=\sqrt{\mathfrak{q} \mathcal{O}_{x}}$. Then there is a integer $k \in \mathbb{N}$ such that $\left(\sqrt{\mathfrak{q} \mathcal{O}_{x}}\right)^{k} \subset \mathfrak{q} \mathcal{O}_{x}$. For any $f \in \mathcal{J}(\mathcal{Z}(\mathfrak{q}))$, its germ $f_{x}$ vanishes on $\mathcal{Z}\left(\mathfrak{q} \mathcal{O}_{x}\right)$, thus $f_{x}^{k} \in \mathfrak{q} \mathcal{O}_{x}$ and Lemma 2.1 ensures that $f^{k} \in \mathfrak{q}$.

Now we prove two slight improvements of Lemma 2.1 and Theorem 2.2 (Lemma 2.3 and Corollary 2.4 below) that will be useful in the sequel.

Lemma 2.3. Let $\mathfrak{q} \subset \mathcal{O}(X)$ be a saturated primary ideal and let $x \in \mathcal{Z}(\mathfrak{q})$. Then, a function $f \in \mathcal{O}(X)$ is in $\sqrt{\mathfrak{q}}$ if and only if its germ $f_{x}$ belongs to an associated prime of $\mathfrak{q} \mathcal{O}_{X, x}$.

Proof. The "only if" implication is clear. For the converse, consider a primary decomposition for the ideal $\mathfrak{q} \mathcal{O}_{X, x}, \mathfrak{q} \mathcal{O}_{X, x}=\mathfrak{q}_{1, x} \cap \cdots \cap \mathfrak{q}_{n, x}$. By hypothesis we can assume that the germ $f_{x}$ is in $\sqrt{\mathfrak{q}_{1, x}}$.

Now, if $n=1$ and $\mathfrak{q} \mathcal{O}_{X, x}=\mathfrak{q}_{1, x}$ let us take $\xi_{x}=1$, otherwise let us take a germ $\xi_{x} \in \bigcap_{i>1} \mathfrak{q}_{i, x} \backslash \mathfrak{q}_{1, x}$. It exists since the decomposition is irreducible. Thus, we have $\xi_{x} f_{x}^{k} \in \mathfrak{q} \mathcal{O}_{X, x}$ for some integer $k \in \mathbb{N}$. Hence, we get that $\xi_{x}$ is in the stalk at $x$ of the sheaf $\mathcal{F}=\left(\mathfrak{q}: f^{k}\right)$. Since $\xi_{x} \notin \mathfrak{q} \mathcal{O}_{X, x}$, there exists at least one global section, say $g \in H^{0}(X, \mathcal{F})$ such that $g \notin \mathfrak{q}$. Since $\mathfrak{q}$ is a saturated ideal this means that $g f^{k} \in \mathfrak{q}$ and, being $\mathfrak{q}$ primary, this implies that a power of $f$ is in $\mathfrak{q}$, as wanted.

Corollary 2.4. Let $\mathfrak{q} \subset \mathcal{O}(X)$ be a saturated primary ideal and let $x \in \mathcal{Z}(\mathfrak{q})$. Assume that an associated prime $\mathfrak{p}_{x} \subset \mathcal{O}_{x}$ of $\mathfrak{q} \mathcal{O}_{X, x}$ has the zero property, meaning by this that $\mathcal{J}\left(\mathcal{Z}\left(\mathfrak{p}_{x}\right)\right)=\mathfrak{p}_{x}$. Then, $\mathcal{J}(\mathcal{Z}(\mathfrak{q}))=\sqrt{\mathfrak{q}}$.

Proof. As before, consider a primary decomposition for the ideal $\mathfrak{q} \mathcal{O}_{X, x}$. We can write $\mathfrak{q} \mathcal{O}_{X, x}=\mathfrak{q}_{1, x} \cap \cdots \cap \mathfrak{q}_{n, x}$ with $\sqrt{\mathfrak{q}_{1, x}}=\mathfrak{p}_{x}$ and $\mathcal{J}\left(\mathcal{Z}\left(\mathfrak{q}_{1, x}\right)\right)=$ $\mathcal{J}\left(\mathcal{Z}\left(\mathfrak{p}_{x}\right)\right)=\mathfrak{p}_{x}$. 
Since the intersection is finite we get

$$
\mathcal{J}\left(\mathcal{Z}\left(\mathfrak{q} \mathcal{O}_{x}\right)\right)=\bigcap_{i=1}^{n} \mathcal{J}\left(\mathcal{Z}\left(\mathfrak{q}_{i, x}\right)\right) \subset \mathcal{J}\left(\mathcal{Z}\left(\mathfrak{q}_{1, x}\right)\right)=\mathfrak{p}_{x}
$$

Hence, for any $f \in \mathcal{J}(\mathcal{Z}(\mathfrak{q}))$ we have that $f_{x} \in \mathfrak{p}_{x}$ and Lemma 2.3 applies.

When dealing with general saturated ideals the main property is the existence of a locally finite primary decomposition. Before doing that, we need to introduce more terminology.

Let $\left\{\mathfrak{a}_{i}\right\}_{i \in I} \subset \mathcal{O}(X)$ be a family of ideals. We say that the family $\left\{\mathfrak{a}_{i}\right\}_{i \in I}$ is locally finite if the family of their zero sets $\left\{\boldsymbol{Z}\left(\mathfrak{a}_{i}\right)\right\}_{i \in I}$ is locally finite.

A decomposition $\mathfrak{a}=\bigcap_{i \in I} \mathfrak{a}_{i}$ is said irreducible if for each $K \subsetneq I, \mathfrak{a} \neq$ $\cap_{i \in K} \mathfrak{a}_{i}$.

Definition 2.5. A locally finite decomposition $\mathfrak{a}=\bigcap_{i} \mathfrak{q}_{i}$ where the $\left\{\mathfrak{q}_{i}\right\}_{i \in I}$ are a family of saturated primary ideals is said a normal primary decomposition (shortly a primary decomposition) if it is irreducible and the associated primes $\sqrt{\mathfrak{q}_{i}}$ are pairwise distinct.

As usual, a primary ideal $\mathfrak{q}_{h} \in\left\{\mathfrak{q}_{i}\right\}_{i \in I}$ is called an isolated primary component if $\sqrt{\mathfrak{q}_{h}}$ is minimal among the primes $\left\{\sqrt{\mathfrak{q}_{i}}\right\}_{i \in I}$. Otherwise it is an immersed primary component.

Next proposition concernes the existence of primary decompositions and can be found in [13, Theorem 2.3.6].

Proposition 2.6. Any saturated ideal $\mathfrak{a} \subset \mathcal{O}(X)$ admits a normal primary decomposition, $\mathfrak{a}=\bigcap_{i} \mathfrak{q}_{i}$, such that all $\mathfrak{q}_{i}$ are saturated and $\boldsymbol{Z}(\mathfrak{a})=\cup_{i} \mathcal{Z}\left(\mathfrak{q}_{i}\right)$.

As it happens in noetherian rings some properties of uniqueness for the primary decomposition hold (Proposition 2.8). We recall for the sake of the reader [13, Lemma 2.2.10].

Lemma 2.7. Let $\left\{\mathfrak{q}_{i}\right\}_{i \in I} \subset \mathcal{O}(X)$ be a locally finite family of saturated primary ideals and let $\mathfrak{p} \subset \mathcal{O}(X)$ be a prime saturated ideal such that $\bigcap_{i} \mathfrak{q}_{i} \subset \mathfrak{p}$. Then there exists $i \in I$ such that $\mathfrak{q}_{i} \subset \mathfrak{p}$.

Proof. By Lemma 2.1 we have to prove that there exists $i \in I$ such that the germ at $x$ of each $f \in \mathfrak{q}_{i}, f_{x}$, is in $\mathfrak{p} \mathcal{O}_{x}$.

Since the $\mathfrak{q}_{i}$ 's, and then their intersection $\bigcap_{i} \mathfrak{q}_{i}$, are saturated ideals, we have the following:

$$
H^{0}\left(X,\left(\bigcap_{i} \mathfrak{q}_{i}\right) \mathcal{O}\right)=\bigcap_{i} \mathfrak{q}_{i}=\bigcap_{i} H^{0}\left(X, \mathfrak{q}_{i} \mathcal{O}\right)=H^{0}\left(X, \bigcap_{i}\left(\mathfrak{q}_{i} \mathcal{O}\right)\right) .
$$


Then, for a point $x \in \mathcal{Z}(\mathfrak{p})$, we get

$$
\left(\bigcap_{i} \mathfrak{q}_{i}\right) \mathcal{O}_{x}=\bigcap_{i}\left(\mathfrak{q}_{i} \mathcal{O}_{x}\right)=\bigcap_{i \in K}\left(\mathfrak{q}_{i} \mathcal{O}_{x}\right)
$$

for some finite set $K \subset I$. Note that such a $K$ exists since the family $\left\{\mathfrak{q}_{i}\right\}_{i}$ is locally finite.

Now, proceeding by contradiction, we assume that, for each $i \in K$ we can find $f_{i} \in \mathfrak{q}_{i}$ such that $f_{i, x} \notin \mathfrak{p} \mathcal{O}_{x}$, that is, $f_{i} \notin \mathfrak{p}$. Since $\prod_{i \in K} f_{i, x} \in$ $\left(\bigcap_{i} \mathfrak{q}_{i}\right) \mathcal{O}_{x} \subset \mathfrak{p} \mathcal{O}_{x}$, we have that $\prod_{i \in K} f_{i} \in \mathfrak{p}$ by Lemma 2.1, contradiction.

Proposition 2.8. Let $\mathfrak{a}=\bigcap_{i} \mathfrak{q}_{i}$ be a primary decomposition for a saturated ideal $\mathfrak{a} \subset \mathcal{O}(X)$. Then, the prime ideals $\mathfrak{p}_{i}=\sqrt{\mathfrak{q}_{i}}$ and the isolated primary components are uniquely determined by $\mathfrak{a}$, that is, they do not depend on the decomposition.

Proof. Fix $i \in I$, since the decomposition is irreducible, there exists $h_{i} \in$ $\bigcap_{j \neq i} \mathfrak{q}_{j} \backslash \mathfrak{q}_{i}$ and then $\sqrt{\left(\mathfrak{a}: h_{i}\right)}=\mathfrak{p}_{i}$. Notice that $\sqrt{\left(\mathfrak{a}: h_{i}\right)}$ is saturated since its zero set is nonempty. Now, let $h \in H^{0}\left(X, \mathcal{O}_{X}\right)$ be such that $\sqrt{(\mathfrak{a}: h)}$ is prime and saturated. We have that

$$
\bigcap_{i \mid h \notin \mathfrak{q}_{i}} \mathfrak{q}_{i} \subset(\mathfrak{a}: h) \subset \sqrt{(\mathfrak{a}: h)} .
$$

By Lemma 2.7, there exists $i$ with $h \notin \mathfrak{q}_{i}$ such that $\mathfrak{q}_{i} \subset \sqrt{(\mathfrak{a}: h)}$. On the other hand, $\sqrt{(\mathfrak{a}: h)} \subset \sqrt{\mathfrak{q}_{i}}$ since $h \notin \mathfrak{q}_{i}$, hence, $\sqrt{(\mathfrak{a}: h)}=\sqrt{\mathfrak{q}_{i}}$.

This proves that the saturated prime ideals $\left\{\mathfrak{p}_{i}\right\}_{i \in I}$ are exactly the saturated prime ideals in $\{\sqrt{(\mathfrak{a}: h)} \mid h \in \mathcal{O}(X)\}$. Now, let $\mathfrak{q}_{i}$ be a isolated primary component. We consider the multiplicative set $S=\mathcal{O}(X) \backslash \mathfrak{p}_{i}$. Since any ideal $\mathfrak{p}_{j}, j \neq i$, intersects $S$, the ideal $\mathfrak{q}_{i}$ coincides with the contraction to $\mathcal{O}(X)$ of the ideal $S^{-1}(\mathfrak{a}) \subset S^{-1}(\mathcal{O}(X))$. Indeed,

$$
S^{-1}(\mathfrak{a})^{c}=\left\{f \in \mathcal{O}(X) \mid \frac{f}{1} \in S^{-1}(\mathfrak{a})\right\} .
$$

Take $f \in S^{-1}(\mathfrak{a})^{c}$. Then there exists $s \in S$ such that $s f \in \mathfrak{a}$ for the general definition of $S^{-1}$. In particular, $s f \in \mathfrak{q}_{i}$ with $s \notin \sqrt{\mathfrak{q}_{i}}$. Hence, $f \in \mathfrak{q}_{i}$.

Finally, to check that $\mathfrak{q}_{i} \subset S^{-1}(\mathfrak{a})^{c}$, note that for any $s \in \cap_{j \neq i} \mathfrak{q}_{j} \backslash \mathfrak{p}_{i}$ (which exists by Lemma 2.7 and $\mathfrak{q}_{i}$ being isolated) we have $s \mathfrak{q}_{i} \subset \mathfrak{a}$.

Hence, $\mathfrak{q}_{i}$ is determined by the fact that $\mathfrak{p}_{i}$ is minimal among the associated primes and $\mathfrak{q}_{i}=S^{-1}(\mathfrak{a})^{c}$.

Note that, unlike what happens in the complex case, a primary decomposition for a saturated ideal $\mathfrak{a}$ does not give necessarily a geometric description of its zero set $z(\mathfrak{a})$. Firstly because in dimension $>2$ there are primary ideals with reducible zero set, for instance $\left(\left(x^{2}-y^{2}\right)^{2}+z^{2}\right) \subset \mathcal{O}\left(\mathbb{R}^{3}\right)$. 
On the other hand because, already in dimension 2, there are distinct prime ideals with the same zero set. For instance $\left(x^{2}+y^{2}\right)$ and $\left(x^{2}+2 y^{2}\right)$ in the ring $\mathcal{O}\left(\mathbb{R}^{2}\right)$.

Proposition 2.9. Let $\mathfrak{a} \subset \mathcal{O}(X)$ be a saturated ideal and let $\mathfrak{a}=\bigcap_{i} \mathfrak{q}_{i}$ be a primary decomposition. Then,

1. If $\mathfrak{a}$ is an $\infty$-real (resp. real or radical) ideal, then every $\mathfrak{q}_{i}$ is an $\infty$-real prime (resp. real prime or prime) ideal. In particolar, the decomposition is unique.

2. If every $\mathfrak{q}_{i}$ has the zero property then the same holds for $\mathfrak{a}$.

Proof. We first prove that if $\mathfrak{a}$ is a radical ideal (whence if $\mathfrak{a}$ is $\infty$-real, or real) then for every $i$ we can find $h_{i} \in \mathcal{O}(X)$ such that $h_{i} \in \bigcap_{j \neq i} \mathfrak{q}_{j} \backslash \sqrt{\mathfrak{q}_{i}}$.

Indeed, assume by contradiction that $\bigcap_{j \neq i} \mathfrak{q}_{j} \subset \sqrt{\mathfrak{q}_{i}}$, then the following inclusions hold:

$$
\bigcap_{j \neq i} \mathfrak{q}_{j} \subset \bigcap_{j \neq i} \mathfrak{q}_{j} \cap \sqrt{\mathfrak{q}_{i}} \subset \sqrt{\bigcap_{j} \mathfrak{q}_{j}}=\sqrt{\mathfrak{a}}=\mathfrak{a} .
$$

Then, $\mathfrak{a}=\bigcap_{j \neq i} \mathfrak{q}_{j}$ and this is impossible, the decomposition being irreducible.

We prove now that if $\mathfrak{a}$ is a radical ideal then every $\mathfrak{q}_{i}$ has to be prime. It is enough to prove that every $\mathfrak{q}_{i}$ is also radical. Fix $i$ and assume that $f^{n} \in \mathfrak{q}_{i}$ for some $n \in \mathbb{N}$. We have to prove that $f \in \mathfrak{q}_{i}$. Now, $h_{i} f^{n} \in \mathfrak{a}$ and a fortiori $\left(h_{i} f\right)^{n} \in \mathfrak{a}$. But $\mathfrak{a}$ is a radical ideal, hence we get $h_{i} f \in \mathfrak{a}$ and then $h_{i} f \in \mathfrak{q}_{i}$. Since $\mathfrak{q}_{i}$ is a primary ideal and $h_{i} \notin \sqrt{\mathfrak{q}_{i}}$ we get that $f \in \mathfrak{q}_{i}$, as wanted.

Now we assume that $\mathfrak{a}$ is also $\infty$-real (resp. real). Fix $i$ and assume that $\sum_{m} f_{m}^{2} \in \mathfrak{q}_{i}$. Take $h_{i} \in \mathcal{O}(X)$ as above, then $h_{i}^{2}\left(\sum_{m} f_{m}^{2}\right)=\sum_{m}\left(h_{i} f_{m}\right)^{2} \in \mathfrak{a}$. In particular each $h_{i} f_{m} \in \mathfrak{a} \subset \mathfrak{q}_{i}$ and then, arguing as above, we get that $f_{m} \in \mathfrak{q}_{i}$ for each $m$.

The uniqueness follows from 2.8.

The second assertion of the statement is a consequence of $2.6: \mathcal{J}(\mathcal{Z}(\mathfrak{a}))=$ $\bigcap_{i} \mathcal{J}\left(\mathcal{Z}\left(\mathfrak{q}_{i}\right)\right)=\bigcap_{i} \mathfrak{q}_{i}=\mathfrak{a}$.

\section{A Nullstellensatz for real coherent surfaces}

The aim of this Section is to prove Theorem 1.1. The proof will be obtained as a consequence of two results that do not need any hypothesis on the dimension.

The first one, Theorem 3.2, considers primary ideals with zero set of codimension one. In some sense, such ideals behave especially well because 
sums of squares are not deeply involved and 2.4 applies. The second one, Theorem 3.5, concerns saturated ideals with compact zero set.

Lemma 3.1. Let $\mathfrak{a} \subset \mathcal{O}_{n}$ be an ideal, then

- $\operatorname{ht}(\mathfrak{a}) \leq n-\operatorname{dim} Z(\mathfrak{a})$ or equivalently $\operatorname{dim}\left(\mathcal{O}_{n} / \mathfrak{a}\right) \geq \operatorname{dim} Z(\mathfrak{a})$

- if $\mathfrak{a}$ is a prime ideal and $\operatorname{ht}(\mathfrak{a})=n-\operatorname{dim} Z(\mathfrak{a})$, then $\mathcal{J}(\mathcal{Z}(\mathfrak{a}))=\mathfrak{a}$.

Proof. The real Nullstellensatz for the regular ring $\mathcal{O}_{n}$ gives us a bijective correspondence beetwen analytic set germs of codimension $p$ and real prime ideals of height $p$, cf. for instance [6, VIII.2.1].

Then, the first assertion follows from the fact that $\mathfrak{a} \subset \mathcal{J}(\mathcal{Z}(\mathfrak{a}))$ and then $\operatorname{ht}(\mathfrak{a}) \leq \operatorname{ht}(\mathcal{J}(\mathcal{Z}(\mathfrak{a})))=n-\operatorname{dim} \mathcal{Z}(\mathfrak{a})$.

For the second assertion, if $\mathfrak{a}$ were a prime ideal strictly contained in $\mathcal{J}(\mathcal{Z}(\mathfrak{a}))$, then we would achieve a contradiction since we would have $\operatorname{ht}(\mathfrak{a})<$ $\operatorname{ht}(\mathcal{J}(\mathcal{Z}(\mathfrak{a})))=\min \{\operatorname{ht}(\mathfrak{p}) \mid \mathfrak{p}$ is a prime ideal containing $\mathcal{J}(\mathcal{Z}(\mathfrak{a}))\}$.

Theorem 3.2. Let $X \subset \mathbb{R}^{N}$ be a real coherent analytic space and let $\mathfrak{q} \subset$ $\mathcal{O}(X)$ be a saturated primary ideal with zero set of codimension 1 in $X$. Then, $\mathcal{J}(\mathcal{Z}(\mathfrak{q}))=\sqrt{\mathfrak{q}}$. In particular, $\sqrt{\mathfrak{q}}$ is a real prime ideal.

Proof. We set $n=\operatorname{dim} X$. Fix a point $x \in \mathcal{Z}(\mathfrak{q})$ such that $\operatorname{dim} z\left(\mathfrak{q} \mathcal{O}_{X, x}\right)=$ $n-1$. Such a point exists by hypothesis. Consider a primary decomposition for $\mathfrak{q} \mathcal{O}_{X, x}$. We can write

$$
\mathfrak{q} \mathcal{O}_{X, x}=\bigcap_{k \in K_{x}} \mathfrak{q}_{k, x} \cap \bigcap_{s \in S_{x}} \mathfrak{q}_{s, x}
$$

where $\operatorname{dim} \mathcal{Z}\left(\mathfrak{q}_{k, x}\right)=n-1$ for any $k \in K_{x}$ and $\operatorname{dim} \mathcal{Z}\left(\mathfrak{q}_{s, x}\right)<n-1$ for any $s \in S_{x}$.

By hypothesis $K_{x} \neq \emptyset$ and for any $k \in K_{x}$ the ideal $\sqrt{\mathfrak{q}_{k, x}}$ is prime with zero set of codimension 1. Fix $k \in K_{x}$; since $X$ is coherent we have

$$
\mathcal{O}_{X, x}=\mathcal{O}_{\mathbb{R}^{N}, x} / \mathcal{J}(X)
$$

and we can choose a prime ideal $\mathfrak{p} \subset \mathcal{O}_{\mathbb{R}^{N}, x}$ such that

$$
\mathcal{J}\left(X_{x}\right) \subset \mathfrak{p} \subset \mathcal{O}_{\mathbb{R}^{N}, x} \quad \text { and } \quad \mathfrak{p} / \mathcal{J}\left(X_{x}\right)=\sqrt{\mathfrak{q}_{k, x}} .
$$

By 2.4 we must prove that $\mathfrak{p}$ (equivalently $\sqrt{\mathfrak{q}_{k, x}}$ ) is a real ideal.

Let $X_{x}=X_{1} \cup \cdots \cup X_{k}$ be a decomposition into irreducible components for the set germ of $X_{x}$ at $x$. Then, $\mathcal{J}\left(X_{x}\right)=\mathfrak{J}\left(X_{1}\right) \cap \cdots \cap \mathcal{J}\left(X_{k}\right)$ is a primary decomposition for the real ideal $\mathcal{J}\left(X_{x}\right)$. In particular, $\mathfrak{p}$ contains the ideal of an irreducible component, say $\mathcal{J}\left(X_{1}\right) \subset \mathfrak{p}$, because $\mathfrak{p}$ contains $\mathcal{J}\left(X_{x}\right)$. 
Now,

$$
\begin{array}{r}
n=\operatorname{dim} X_{x}=\max \left\{\operatorname{dim} X_{i} \mid i=1, \ldots, k\right\} \\
N-n=\operatorname{ht}\left(\mathcal{J}\left(X_{x}\right)\right)=\min \left\{\operatorname{ht}\left(\mathcal{J}\left(X_{i}\right)\right) \mid i=1, \ldots, k\right\} .
\end{array}
$$

In particular,

$$
\operatorname{ht}(\mathfrak{p}) \geq \operatorname{ht}\left(\mathcal{J}\left(X_{1}\right)\right) \geq N-n .
$$

On the other hand, by 3.1 we have $h t(\mathfrak{p}) \leq N-(n-1)$. Then we must consider two cases: either ht $(\mathfrak{p})=N-(n-1)$ or ht $(\mathfrak{p})=N-n$.

In the first case, 3.1 ensures that $\mathfrak{p}$ has the zero property. In the second case, we have $\operatorname{ht}\left(\mathcal{J}\left(X_{1}\right)\right)=N-n$ and then $\mathcal{J}\left(X_{1}\right)=\mathfrak{p}$ because they are prime ideals and have the same height. In both cases the ideal $\mathfrak{p}$ is real, as we wanted.

As an immediate corollary of 2.9 and 3.2 we get:

Corollary 3.3. If $\mathfrak{a}$ is a radical ideal whose normal primary decomposition $\mathfrak{a}=\bigcap \mathfrak{q}_{i}$ is such that $\mathcal{Z}\left(\mathfrak{q}_{i}\right)$ has codimension 1 for all $i$, then $\mathfrak{a}$ has the zero property. In particular, $\mathfrak{a}$ is also a real ideal.

Remark 3.4. (a) Notice that a primary ideal $\mathfrak{q} \subset \mathcal{O}(X)$ verifying the hypothesis of 3.2 has irreducible zero set $Z(\mathfrak{q})$ since $\mathcal{J}(\mathcal{Z}(\mathfrak{q}))$ is a prime ideal.

(b) If $X$ is a real analytic manifold with $H^{1}(X, \mathbb{Z} / 2 \mathbb{Z})=0$ then an ideal $\mathfrak{q}$ verifying the hypothesis of 3.2 is principal. Indeed, $\mathcal{J}(\mathcal{Z}(\mathfrak{q}))$ is a principal ideal, being the ideal of a hypersurface, and this implies that $\mathfrak{q}$ is also principal (see 4.2 below).

(c) Theorem 3.2 was already known for a real prime ideal $\mathfrak{p}$ in a real analytic manifold $X$, see [4] and Theorem 4.7, Remark 4.8 below.

Proposition 3.5. Let $X \subset \mathbb{R}^{n}$ be a real coherent analytic set and let $\mathfrak{a} \subset$ $\mathcal{O}(X)$ be a saturated ideal with compact zero set. Then, $\mathcal{J}(\mathcal{Z}(\mathfrak{a}))=\sqrt[r]{\mathfrak{a}}$.

Proof. This fact is proved in [6] for a finitely generated ideal with compact zero set. Thus, it is enough to prove that a saturated ideal with compact zero set is always finitely generated. Indeed, take $x \in Z(\mathfrak{a})$. Since $\mathfrak{a} \mathcal{O}$ is a coherent sheaf, there exist $s_{x, 1}, \ldots, s_{x, p(x)} \in H^{0}(X, \mathfrak{a} \mathcal{O})=\mathfrak{a}$ and a neighbourhood $U(x)$ of $x$ such that $\mathfrak{a} \mathcal{O}_{y}=\left(s_{x, 1}, \ldots, s_{x, p(x)}\right) \mathcal{O}_{y}$ for any $y \in U(x)$. Now, since $\bigcup_{x \in \mathcal{Z}(\mathfrak{a})} U(x)$ is an open covering for $\mathcal{Z}(\mathfrak{a})$ and $Z(\mathfrak{a})$ is compact we can take a finite subcovering, $U\left(x_{1}\right), \ldots, U\left(x_{n}\right)$. We have that

$$
\mathfrak{a} \mathcal{O}_{y}=\left\{\begin{array}{cc}
\left(s_{x_{i}, 1}, \ldots, s_{x_{i}, p\left(x_{i}\right)}\right) \mathcal{O}_{y} & \text { if } x \in U\left(x_{i}\right) \\
\mathcal{O}_{x} & \text { if } x \notin \mathcal{Z}(\mathfrak{a})
\end{array}\right.
$$

By [12], there exists a finite number of global sections $s_{1}, \ldots, s_{t} \in \mathfrak{a}=$ $H^{0}(X, \mathfrak{a} \mathcal{O})$ that generate the stalk at each point. Hence, $\mathfrak{a}$ is generated by $s_{1}, \ldots, s_{t}$ as wanted. 
Proof of Theorem 1.1. By 2.9 we can assume $\mathfrak{a}$ to be a real saturated prime ideal. Being $\operatorname{dim} X=2$, either $\operatorname{dim} z(\mathfrak{a})=1$ or $\operatorname{dim} z(\mathfrak{a})=0$, unless $\mathfrak{a}$ is trivial. In the first case Theorem 3.2 applies. In the second case remark that the zero set of a primary saturated ideal is a connected set; hence $\mathcal{Z}(\mathfrak{a})$ is a point and Theorem 3.5 applies.

\section{Nullstellensatz and Hilbert 17 th problem for $\mathbb{R}^{3}$}

The aim of this section is to prove Theorem 1.3. As a consequence we will compare the Nullstellensatz problem with the Hilbert 17th one. As remarked in the Introduction, we will see that these two classical problems have the same kind of obstruction concerning irreducible special functions.

To give a precise definition we need to recall the classical construction of the (complex) irreducible factors of a global analytic function $f \in \mathcal{O}\left(\mathbb{R}^{3}\right)$ (see for instance [15, Section 2]).

Let $f$ be an analytic function in $\mathcal{O}\left(\mathbb{R}^{3}\right)$. There exist:

- An open invariant Stein neighbourhood $\mathcal{U}$ of $\mathbb{R}^{3}$ in $\mathbb{C}^{3}$ such that $\mathbb{R}^{3}$ is a deformation retract of $\mathcal{U}$;

- A holomorphic extension $F$ of $f$ to $\mathfrak{U}$;

- Holomorphic functions $H_{j}: \mathcal{U} \rightarrow \mathbb{C}, j \in J$, such that $\left\{S_{j}=H_{j}^{-1}(0)_{\mathbb{R}^{3}}\right\}_{j}$ are the (complex) irreducible components of the germ $F^{-1}(0)_{\mathbb{R}^{3}}$ and $H_{j}$ generates the ideal of $H_{j}^{-1}(0)$. Furthermore, if $S_{j}$ is invariant (by conjugation) we may assume that $H_{j}$ is also invariant, hence $h_{j}=\left.H_{j}\right|_{\mathbb{R}^{3}}$ defines a real analytic function. Otherwise, there exists an index $j^{\prime} \neq j$ such that $S_{j}$ and $S_{j^{\prime}}$ are conjugated and we may assume that $H_{j^{\prime}}(z)=\overline{H_{j} \circ \sigma(z)}$, hence $h_{j j^{\prime}}=\left.H_{j} H_{j^{\prime}}\right|_{\mathbb{R}^{3}}$ defines a real analytic function which is a sum of two squares.

Now, the holomorphic function germs $H_{j, \mathbb{R}^{3}}$ are uniquely determined by $f=\left.F\right|_{\mathbb{R}^{3}}$. Thus, we will say that $\left\{H_{j, \mathbb{R}^{3}}\right\}$ are the (complex) irreducible factors of $f$.

Definition 4.1. (a) We say that $H_{j, \mathbb{R}^{3}}$ is a special factor of $f$ if it is invariant by conjugation, the dimension of the real analytic set $H_{j}^{-1}(0) \cap \mathbb{R}^{3}$ is 1 and $H_{j}$ divides $F$ with odd multiplicity.

(b) If $f$ has only one irreducible factor and it is special, we say that $f$ is a special analytic function.

Remark that an analytic function $f \in \mathcal{O}\left(\mathbb{R}^{3}\right)$ is said to be irreducible if it cannot be written as the product of two analytic functions with nonempty zero set; [4, Lemma 4.5] ensures that any irreducible function generates a prime ideal. 
If $f$ is irreducible, then either $f$ has one irreducible factor that is invariant by conjugation, or $f$ has two irreducible factors which are conjugated. In the first case $F$ is irreducible, in the second one $F$ is reducible and $f$, up to a unit, is a sum of two squares.

The main result in [15] states that every nonnegative function $f: \mathbb{R}^{3} \rightarrow \mathbb{R}$ is an infinite sum of squares of meromorphic functions if and only if this is true for every nonnegative special irreducible function. The author also considers analogous properties involving only finite sums of squares but the problem is quite more delicate and only partial results are proved, see for instance [15, Theorem 1.6].

In the spirit of these results we will prove Theorem 1.3. First of all, we reduce to consider principal ideals (Theorem 4.3), then principal ideals generated by special functions (Proposition 4.4).

Lemma 4.2. Let $\mathfrak{q} \subset \mathcal{O}\left(\mathbb{R}^{n}\right)$ be a primary saturated ideal. Then, $\mathfrak{q}$ is a principal ideal if and only if $\sqrt{\mathfrak{q}}$ is a principal ideal.

Proof. For the "if" implication, assume that $\sqrt{\mathfrak{q}}$ is a principal ideal generated by $f \in \mathcal{O}\left(\mathbb{R}^{n}\right)$, then it is easy to see that $\mathfrak{q}$ is generated by $f^{k}$ where $k=\min \left\{m \in \mathbb{N} \mid f^{m} \in \mathfrak{q}\right\}$. Note moreover that $f$ has to be irreducible.

Now we prove the "only if" implication. Assume that $\mathfrak{q}$ is generated by $f \in O\left(\mathbb{R}^{n}\right)$. By [11], there exists $h \in \mathcal{O}\left(\mathbb{R}^{n}\right)$ such that $\left(h_{x}\right) \mathcal{O}_{x}=\sqrt{\left(f_{x}\right) \mathcal{O}_{x}}$ for each point $x \in \mathbb{R}^{n}$.

We claim that $\sqrt{\mathfrak{q}}=(h)$. On the one hand, if $g \in \sqrt{\mathfrak{q}}=\sqrt{(f)}$, then the germ $g_{x}$ is in $\sqrt{\left(f_{x}\right) \mathcal{O}_{x}}=\left(h_{x}\right)$ for each $x \in \mathbb{R}^{n}$ and then $g \in(h)$.

On the other hand, we must prove that $h \in \sqrt{\mathfrak{q}}$. Take a point $y \in Z(\mathfrak{q})$, since $\left(h_{x}\right)=\sqrt{\left(f_{x}\right) \mathcal{O}_{x}}$ for each $x \in \mathbb{R}^{n}$, we can find an integer $m$ such that $h_{y}^{m} \in\left(f_{y}\right)=\mathfrak{q} \mathcal{O}_{y}$. Being $\mathfrak{q}$ a saturated primary ideal, 2.1 ensures that $h^{m} \in \mathfrak{q}$, as we wanted.

Theorem 4.3. Let $\mathfrak{q} \subset \mathcal{O}\left(\mathbb{R}^{3}\right)$ be a saturated primary ideal such that $\operatorname{dim} \mathcal{Z}(\mathfrak{q})=1$. Then, $\mathcal{J}(\mathcal{Z}(\mathfrak{q}))=\sqrt{\mathfrak{q}}$ if and only if $\mathfrak{q}$ is not a principal ideal.

Proof. We first prove the "only if" implication. We proceed by way of contradiction. Assume that $\mathfrak{q}$ is a principal ideal, then $\sqrt{\mathfrak{q}}=\mathfrak{J}(\mathcal{Z}(\mathfrak{q}))$ is also a principal ideal. We achieve a contradiction showing that the ideal of a curve in $\mathbb{R}^{3}$ cannot be principal.

Indeed, if $x \in \mathcal{Z}(\mathfrak{q})$ then $\mathcal{J}(\mathcal{Z}(\mathfrak{q})) \mathcal{O}_{x}=\mathcal{J}\left(\mathcal{Z}\left(\mathfrak{q} \mathcal{O}_{x}\right)\right)$ since curves are coherent.

Now, if $\mathcal{J}(\mathcal{Z}(\mathfrak{q}))$ is a principal ideal, $\mathcal{J}(\mathcal{Z}(\mathfrak{q})) \mathcal{O}_{x}$ is also a principal ideal and, being $\mathcal{O}_{x}$ a unique factorization domain, this implies that its height is 1 , while the height of the ideal $\mathcal{J}\left(\mathcal{Z}\left(\mathfrak{q} \mathcal{O}_{x}\right)\right)$ should be 2 since $Z(\mathfrak{q})$ is connected and hence pure dimensional. 
Let us prove the "if" implication. For any $x \in \mathcal{Z}(\mathfrak{q})$ consider a primary decomposition for the ideal $\mathfrak{q} \mathcal{O}_{x} \subset \mathcal{O}_{x}$. We can write

$$
\mathfrak{q} \mathcal{O}_{x}=\bigcap_{k \in K_{x}} \mathfrak{q}_{k, x} \cap \bigcap_{j \in J_{x}} \mathfrak{q}_{j, x} \cap \bigcap_{s \in S_{x}} \mathfrak{q}_{s, x}
$$

where the sets $K_{x}, J_{x}, S_{x}$ are finite, possibly empty, and satisfy the following properties:

(1) If $k \in K_{x}$, then $\operatorname{dim} z\left(\mathfrak{q}_{k, x}\right)=1$ and $\operatorname{ht}\left(\mathfrak{q}_{k, x}\right)=2$

(2) If $j \in J_{x}$, then $\operatorname{dim} z\left(\mathfrak{q}_{j, x}\right)=1$ and $\operatorname{ht}\left(\mathfrak{q}_{j, x}\right)=1$

(3) If $s \in S_{x}$, then $z\left(\mathfrak{q}_{s, x}\right)=\{x\}$. In this case ht $\left(\mathfrak{q}_{s, x}\right)$ can be 1,2 or also 3 .

Every $\mathfrak{q}_{k, x}$ is a primary ideal of height 2 whose zero set has codimension 2 , hence by 3.1

$$
\mathcal{J}\left(\mathcal{Z}\left(\mathfrak{q}_{k, x}\right)\right)=\mathcal{J}\left(\mathcal{Z}\left(\sqrt{\mathfrak{q}_{k, x}}\right)\right)=\sqrt{\mathfrak{q}_{k, x}} .
$$

Then, by 2.4 , we are reduced to prove that there exists a point $x \in z(\mathfrak{q})$ where $K_{x} \neq \emptyset$.

We claim that $S_{x}$ is empty. Otherwise, assume that there exists $s \in S_{x}$ and consider the sheaf of ideals defined by

$$
\mathcal{F}_{y}=\left\{\begin{array}{cc}
\mathfrak{q}_{s, x} & \text { if } y=x \\
\mathcal{O}_{y} & \text { if } y \neq x
\end{array}\right.
$$

Then, there are global sections $\eta_{1}, \ldots, \eta_{s} \in \mathcal{O}\left(\mathbb{R}^{3}\right)$ that generate the stalk at each point, that is

$$
\mathfrak{q}_{s, x}=\left(\eta_{1}, \ldots, \eta_{s}\right)_{x} \quad \text { and } \quad z\left(\eta_{1}^{2}+\cdots+\eta_{s}^{2}\right)=\{x\} .
$$

On the one hand $\eta=\eta_{1}^{2}+\cdots+\eta_{s}^{2}$ is in $\sqrt{\mathfrak{q}}$, because of 2.3 ; on the other hand, no power of $\eta$ can be in $\mathfrak{q}$ since $\mathcal{Z}(\eta)=\{x\}$, a contradiction.

Thus, at each point $x \in \mathcal{Z}(\mathfrak{q})$ we can write

$$
\mathfrak{q} \mathcal{O}_{x}=\bigcap_{k} \mathfrak{q}_{k, x} \cap \bigcap_{j} \mathfrak{q}_{j, x}=\mathfrak{q}_{1, x} \cap \mathfrak{q}_{2, x}
$$

Now we prove that there exists a point $x \in \mathcal{Z}(\mathfrak{q})$ where $\mathfrak{q} \mathcal{O}_{x} \neq \mathfrak{q}_{2, x}$. Indeed, every $\mathfrak{q}_{j, x}$ is a primary ideal of height one in a unique factorization domain, $\mathcal{O}_{x}$, hence it is principal, namely $\mathfrak{q}_{j, x}=\left(\xi_{j, x}^{r(x)}\right) \mathcal{O}_{x}$ where $\xi_{j, x} \in \mathcal{O}_{x}$ is an irreducible germ. Thus, if $J_{x} \neq \emptyset$ the ideal $\mathfrak{q}_{2, x}$ is a principal ideal, say $\mathfrak{q}_{2, x}=\left(\xi_{x}\right)$. If $\mathfrak{q} \mathcal{O}_{x}=\mathfrak{q}_{2, x}$ for any $x \in \mathfrak{Z}(\mathfrak{q})$, then $\mathfrak{q}$ is locally principal and, because $H^{1}\left(\mathbb{R}^{3}, \mathbb{Z} / 2 \mathbb{Z}\right)=0, \mathfrak{q}$ is a principal ideal, contradiction. 
Proposition 4.4. Let $f \in \mathcal{O}\left(\mathbb{R}^{3}\right)$ be such that $\operatorname{dim} \mathcal{Z}(f)=1$ and the principal saturated ideal $(f) \mathcal{O}\left(\mathbb{R}^{3}\right)$ is primary. Then, up to a unit, either $f$ coincides with $h^{s}$ for some special irreducible function $h \in \mathcal{O}\left(\mathbb{R}^{3}\right)$ and some integer $s \geq 1$, or $f$ coincides with a finite sum of squares.

Proof. Let $h \in \mathcal{O}\left(\mathbb{R}^{3}\right)$ be such that $\sqrt{(f) \mathcal{O}\left(\mathbb{R}^{3}\right)}=(h) \mathcal{O}\left(\mathbb{R}^{3}\right)$. Such an $h$ exists by 4.2 and has to be irreducible since it generates a prime ideal. Then, as in the proof of 4.2 , we get that $(f)=\left(h^{s}\right)$ for $s=\min \left\{n \in \mathbb{N} \mid h^{n} \in(f)\right\}$. This means that $f$ and $h^{s}$ coincide up to a unit.

Now, if $h$ has only one complex irreducible factor that is invariant by conjugation, then $h$ is special since $\operatorname{dim} z(h)=1$, otherwise, up to the sign, $h$ should be a sum of two squares. Then, up to a unit, either $f$ coincides with a power of a special irreducible function or $f$ coincides with a finite sum of squares.

Lemma 4.5. Let $f: \mathbb{R}^{3} \rightarrow \mathbb{R}$ be an infinite (resp. finite) sum of squares of meromorphic functions. Then, the ideal $(f) \mathcal{O}\left(\mathbb{R}^{3}\right)$ is not $\infty$-real (resp. real).

Proof. By hypothesis there exist $h_{0}, h_{1}, \cdots \in \mathcal{O}\left(\mathbb{R}^{3}\right)$ (resp. $h_{0}, h_{1}, \ldots, h_{N} \in$ $\left.\mathcal{O}\left(\mathbb{R}^{3}\right)\right)$ such that $\mathcal{Z}\left(h_{0}\right) \subset \mathcal{Z}(f)$ and $h_{0}^{2} f=\sum_{n \geq 1} h_{n}{ }^{2}$. Let $k \geq 0$ be the maximum integer such that $f^{k}$ divides each $h_{i}$. Hence, we can write

$$
{h_{0}^{\prime 2}}^{2 k+1}=f^{2 k} \sum_{n \geq 1}{h_{n}^{\prime 2}}^{2}
$$

and dividing the equation by $f^{2 k}$ we get

$$
{h_{0}^{\prime 2}}^{2} f=\sum_{n \geq 1}{h_{n}^{\prime 2}}^{2}
$$

If $(f)$ is an $\infty$-real (resp. real) ideal, then $f$ has to divide $h_{n}^{\prime}$ for all $n \geq 1$, and $f$ does not divide $h_{0}^{\prime}$. Then, $f^{2}$ divides the right hand side of the equation, hence $f$ divides $h_{0}^{\prime 2}$, and since $(f)$ is $\infty$-real (real), $f$ divides $h_{0}^{\prime}$, contradiction.

Now we are ready to prove Theorem 1.3.

Proof of Theorem 1.3. The "only if" implication is clear: a special irreducible function can not generate an ideal wirh the zero property since the ideal of a curve in $\mathbb{R}^{3}$ is not a principal ideal. Let us consider the other implication. Let $\mathfrak{a} \subset \mathcal{O}\left(\mathbb{R}^{3}\right)$ be a $\infty$-real (resp. real) saturated ideal, hence it admits a decomposition $\mathfrak{a}=\bigcap_{i} \mathfrak{q}_{i}$ as a locally finite intersection of $\infty$-real (resp. real) prime saturated ideals. 
We have to prove that $\mathcal{J}\left(\mathcal{Z}\left(\mathfrak{q}_{i}\right)\right)=\mathfrak{q}_{i}$. for each $i$. If $\operatorname{dim} \mathcal{Z}\left(\mathfrak{q}_{i}\right)=1$, we are left to prove that $\mathfrak{q}_{i}$ cannot be principal, by 4.3. Now, $\mathfrak{q}_{i}$ being a real ideal, it cannot be generated by a finite sum of squares. Then, by 4.4 , it is generated by a power of a special irreducible function. Moreover, since $\mathfrak{q}_{i}$ is prime, it is generated by a special irreducible function. Then, it cannot be a $\infty$-real (resp. real) ideal by hypothesis, contradiction.

If $\operatorname{dim} \mathcal{Z}\left(\mathfrak{q}_{i}\right)=0$ or 2 , it follows from 3.2 and 3.5 that $\mathcal{J}\left(\mathcal{Z}\left(\mathfrak{q}_{i}\right)\right)=\mathfrak{q}_{i}$. Hence, $\mathcal{J}(\mathcal{Z}(\mathfrak{a}))=\bigcap \mathcal{J}\left(\mathcal{Z}\left(\mathfrak{q}_{i}\right)\right)=\bigcap \mathfrak{q}_{i}=\mathfrak{a}$.

Remark 4.6. In particular, 4.5 and 1.3 ensure that a positive answer to the Hilbert 17 th problem on $\mathbb{R}^{3}$ would imply a solution for the Nullstellensatz problem for $\infty$-real saturated ideals too.

We end this Section describing some results for higher dimension.

Let $X \subset \mathbb{R}^{n}$ be a real analytic manifold and let $\left(\widetilde{X}, \mathcal{O}_{\tilde{X}}\right)$ be a complexification of $X$. Let $\mathfrak{a} \subset \mathcal{O}(X)$ be an ideal. Then, $\mathfrak{a} \otimes_{\mathbb{R}} \mathbb{C} \subset H^{0}\left(X, \mathcal{O}_{\tilde{X}}\right)$ is an ideal that defines the germ of a complex analytic set, $Z\left(\mathfrak{a} \otimes_{\mathbb{R}} \mathbb{C}\right)$, at $X$. Such analytic set can be defined as follows. The germ $z\left(\mathfrak{a} \otimes_{\mathbb{R}} \mathbb{C}\right)_{x}$ at a point $x \in X$ is the germ of the variety determined by the local ideal $\mathfrak{a} \mathcal{O}_{X, x} \otimes_{\mathbb{R}} \mathbb{C}$. Since the sheaf $\mathfrak{a} \mathcal{O}_{X}$ is coherent these germs fit together to give the germ $\mathcal{Z}\left(\mathfrak{a} \otimes_{\mathbb{R}} \mathbb{C}\right)$ at $X$. The following result is proved in [4, Theorem 3.5].

Theorem 4.7. Let $\mathfrak{p} \subset \mathcal{O}(X)$ be a real prime ideal. Assume that there exists a point $x \in X$ such that $\operatorname{dim}_{\mathbb{R}}(\mathcal{Z}(\mathfrak{p}), x)=\operatorname{dim}_{\mathbb{C}}\left(\mathcal{Z}\left(\mathfrak{p} \otimes_{\mathbb{R}} \mathbb{C}\right), x\right)$. Then $\mathfrak{p}$ has the zero property.

Remark 4.8. As remarked in 3.4(c), Proposition 3.2 for a real prime ideal $\mathfrak{p}$ of an analytic manifold $X$ follows straightforwardly from this result.

In what follows, an ideal $\mathfrak{a} \subset \mathcal{O}(X)$ is called a special ideal if it is a prime ideal such that $\mathfrak{a} \otimes_{\mathbb{R}} \mathbb{C}$ is prime and

$$
\operatorname{dim}_{\mathbb{R}}(\mathcal{Z}(\mathfrak{a}), x)<\operatorname{dim}_{\mathbb{C}}\left(Z\left(\mathfrak{a} \otimes_{\mathbb{R}} \mathbb{C}\right), x\right)
$$

for any $x \in \mathcal{Z}(\mathfrak{a})$.

Remark 4.9. Note that the notion of special ideal appears as a natural generalization of the notion of special function. In particular any special irreducible function $f \in \mathcal{O}\left(\mathbb{R}^{3}\right)$ generates a special ideal $(f) \mathcal{O}\left(\mathbb{R}^{3}\right) \subset \mathcal{O}\left(\mathbb{R}^{3}\right)$.

In the spirit of Theorem 1.3 we have the following result:

Theorem 4.10. Any $\infty$-real (resp. real) saturated ideal in $\mathcal{O}(X)$ has the zero property if and only if no special ideal is $\infty$-real (resp. real). 
Proof. For the "only if" part, note that if a saturated ideal $\mathfrak{a}$ has the zero property, then the sheaf $\mathfrak{a} \mathcal{O}$ defines the so called "well reduced structure" on $Z(\mathfrak{a})$, see [17] and [3], and, in particular $\operatorname{dim}_{\mathbb{R}}(Z(\mathfrak{a}), x)=\operatorname{dim}_{\mathbb{C}}\left(Z\left(\mathfrak{a} \otimes_{\mathbb{R}} \mathbb{C}\right), x\right)$ for all $x \notin Y$ where $\operatorname{dim} Y<\operatorname{dim} z(\mathfrak{a})$.

For the "if" part, let $\mathfrak{a}$ be a $\infty$-real (resp. real) saturated ideal and let $\mathfrak{a}=\bigcap \mathfrak{p}_{i}$ be the decomposition of $\mathfrak{a}$ into $\infty$-real (resp. real) saturated prime ideals. Since no $\mathfrak{p}_{i}$ can be special, 4.7 implies that $\mathcal{J}\left(\mathcal{Z}\left(\mathfrak{p}_{i}\right)\right)=\mathfrak{p}_{i}$. Hence, as usual, $\mathcal{J}(\mathcal{Z}(\mathfrak{a}))=\bigcap \mathcal{J}\left(\mathcal{Z}\left(\mathfrak{p}_{i}\right)\right)=\bigcap \mathfrak{a}_{i}=\mathfrak{a}$, and we are done.

\section{References}

[1] Acquistapace, F., Broglia, F., Fernando, J. F. and Ruiz, J. M.: On the finiteness of Pythagoras numbers of real meromorphic functions. Article in press in Bull. Soc. Math. France (2009).

[2] Acquistapace, F., Broglia, F., Fernando, J. F.: On a global analytic Positivstellensatz. Ark. Mat. 47 (2009), no. 1, 13-39.

[3] Acquistapace, F., Broglia, F., Tognoli, A.: Sulla normalizzazione degli spazi analitici reali. Boll. Un. Mat. Ital. (4) 12 (1975), no. 1-2, 26-36.

[4] Adkins, W. A.: A real analytic Nullstellensatz for two dimensional manifolds. Boll. Un. Mat. Ital. B (5) 14 (1977), no. 3, 888-903.

[5] Adkins, W. A. And LeAhy, J. V.: A global real analytic Nullstellensatz. Duke Math. Journal 43 (1976), no. 1, 81-86.

[6] Andradas, C., Bröcker, L. and Ruiz, J. M.: Constructible sets in real geometry. Ergebnisse der Mathematik und ihrer Grenzgebiete (3) 33. Springer-Verlag, Berlin, 1996.

[7] Andradas, C. And Díaz-Cano, A.: Some properties of global semianalytic subsets of coherent surfaces. Illinois J. Math. 48 (2004), no. 2, 519-537.

[8] Atiyah, M. F. and Macdonald, I. G.: Introduction to commutative algebra. Addison-Wesley Publishing Co., Reading, Mass.-London-Don Mills, Ont., 1969.

[9] Bochnak, J., Coste, M. And Roy, M.F.: Real algebraic geometry. Ergebnisse der Mathematik und ihrer Grenzgebiete (3) 36. Springer-Verlag, Berlin, 1998.

[10] Bochnak, J. AND Risler, J. J.: Le théorème des zéros pour les variétés analytiques réelles de dimension 2. Ann. Sci. École Norm. Sup. (4) 8 (1975), no. $3,353-363$.

[11] Cain, B. E.: A two color theorem for analytic maps in $\mathbb{R}^{n}$. Proc. Amer. Math. Soc. 39 (1973), 261-266.

[12] Coen, S.: Sul rango dei fasci coerenti. Boll. Un. Mat. Ital. (3) 22 (1967), 373-382. 
[13] De Bartolomeis, P.: Algebre di Stein nel caso reale. Rend. Accad. Naz. XL (5) 1/2 (1975/76), 105-144 (1977).

[14] De Bartolomeis, P.: Una nota sulla topologia delle algebre reali coerenti. Boll. Un. Mat. Ital. (5) 13A (1976), no. 1, 123-125.

[15] Fernando, J. F.: On the 17th Hilbert Problem for global analytic functions on dimension 3. To appear in Commentarii Math. Helvetici.

[16] Forster, O.: Primärzerlung in Steinschen Algebren. Math. Annalen 154 (1964), 307-329.

[17] Galbiati, M.: Stratifications et ensemble de non-cohérence d'un espace analytique réel. Invent. Math. 34 (1976), no. 2, 113-128.

[18] Galbiati, M. And Tognoli, A.: Alcune proprietà delle varità algebriche reali. Ann. Scuola Norm. Sup. Pisa (3) 27, 359-404 (1973).

[19] Pieroni, F.: A sufficient condition for a real global Nulstellensatz. Preprint.

[20] Ruiz, J.: On Hilbert's 17th problem and real Nullstellensatz for global analytic functions. Math. Z. 190 (1985), no. 3, 447-454.

[21] Ruiz, J.: The basic theory of power series. Advanced Lectures in Mathematicas. Friedr. Vieweg \& Sohn, Braunschweig, 1993.

[22] Siu, Y. T.: Hilbert Nullstellensatz in global complex analytic case. Proc. Amer. Math. Soc. 19 (1968), 296-298.

[23] Tognoli, A.: Proprietà globali degli spazi analitici reali. Ann. Mat. Pura Appl. (4) 75 (1967), 143-218.

Recibido: 21 de mayo de 2007

Revisado: 23 de noviembre de 2007

Fabrizio Broglia

Dipartimento di Matematica

Via Filippo Buonarroti 2

56127 Pisa, Italy

broglia@dm.unipi.it

Federica Pieroni

Dipartimento di Matematica

Via Filippo Buonarroti 2

56127 Pisa, Italy

pieroni@mail.dm.unipi.it

Members of GNSAGA, partially supported by MURST. 